\title{
Hybrid Nonlinear Modeling Using Adaptive Sampling
}

\author{
Paweł Barmuta, Student Member, IEEE, Gustavo Avolio, Member, IEEE, Francesco Ferranti, Member, IEEE, \\ Arkadiusz Lewandowski, Member, IEEE, Luc Knockaert, Senior Member, IEEE, \\ Dominique Schreurs, Fellow, IEEE
}

\begin{abstract}
This paper proposes a direct method for the extraction of empirical-behavioral hybrid models using adaptive sampling. The empirical base is responsible for the functionality over a wide range of variables, especially in the extrapolation range. The behavioral part corrects for the errors of the empirical part in the region of particular interest, thus, it improves the accuracy in the desired region. Employment of response surface methodology and adaptive sampling allows full automation of the hybrid model extraction and assures its compactness. We used this approach to build a hybrid model composed of a robust empirical model available in CAD tools and a Radial Basis Functions interpolation model with Gaussian basis function. We extracted the hybrid model from measurements of a 0.15 $\mu \mathrm{m}$ GaAs HEMT and compared it to the pure behavioral and pure empirical models. The hybrid model yields higher accuracy while maintaining extrapolation capabilities. Additionally, the extraction time of the hybrid model is relatively low and we show that a good accuracy level can be achieved with a small number of measurements.
\end{abstract}

Index Terms-behavioral modeling, response surface, experimental design, active device modeling, adaptive sampling

\section{INTRODUCTION}

$\mathbf{T}$ WO main model types are commonly used for the modeling of microwave transistors: empirical and behavioral models. By accounting for the physical constraints present in the device, empirical models mimic the device behavior over a broad range of various variables, e.g., bias voltages, frequencies, etc. However, user experience and understanding of the physical phenomena are required in order to construct these models. Therefore, empirical models are less general, and their extraction procedures are relatively complex. This

This paper is an expanded version from the IEEE MTT-S International Microwave Symposium, Phoenix, USA, May 17-22, 2015.

This work was supported by the Research Foundation Flanders (FWOVlaanderen) and Hercules Foundation.

P. Barmuta (pbarmuta@stud.elka.pw.edu.pl) and A. Lewandowski are with the Department of Electronics and Information Technology, Warsaw University of Technology, Poland. P. Barmuta was supported by the European Union in the framework of European Social Fund through the Warsaw University of Technology Development Programme. A. Lewandowski was supported by the Polish National Science Center in the framework of project no. 2011/03/D/ST7/01731.

P. Barmuta (pawel.barmuta@esat.kuleuven.be), G. Avolio and D. Schreurs are with the Department of Electrical Engineering, KU Leuven, Belgium. G. Avolio is Post-Doctoral Research Fellow of FWO-Vlaanderen.

F. Ferranti is with the Department of Fundamental Electricity and Instrumentation, Vrije Universiteit Brussel, Belgium. F. Ferranti is a Post-Doctoral Research Fellow of FWO-Vlaanderen.

L. Knockaert is with the Department of Information Technology, Ghent University, Belgium. leads to multiple model modifications and adjustments preceded by thorough measurement investigations [1], [2].

An opposite scenario is related to behavior models. They are not linked to device physics and are usually straightforward to extract. Their generality allows modeling any kind of devices with great accuracy [3]. However, behavioral models are strictly limited by the regions of input variables, for which the models were extracted. In other words, behavioral models perform very well in interpolation tasks, while their accuracy degrades in extrapolation tasks. Lack of prediction capabilities enforces that the modeling and measurements are performed in the same space of input and output variables, which have to be considered during the transistor measurements [4]. This, in turn, entails the need of collecting a very large number of (typically large-signal) measurements [5], [6], which serve in extraction of very complex models [7], [8].

The last problem can be addressed by employment of the response surface methodology [9], [10]. It is based on adaptive sampling, in which consecutive samples (combinations of input variables' values or Large Signal Operating Points (LSOP)) are determined from the previously acquired data. As a result, the amount of information given by each measurement is maximized, and considerably less samples may be required to build an accurate behavioral model [11].

Many works proposed hybrid models combining the advantages of both approaches while overcoming their drawbacks. Some authors propose to improve the accuracy of the empirical models by expanding the number of model coefficients [12]. More degrees of freedom allow achieving a higher level of generality and accuracy. The other way of constructing a hybrid model is to propagate or add the response of one type of model through the response of a second model. This solution is particularly useful when used to combine a behavioral model for the nonlinear transistor core and an empirical model for the parasitic components [3], [13]-[15].

In previous work [16], the robust empirical model available in CAD tools [17] has been used as a reference to assess the performance of the behavioral models extracted with adaptive sampling. Even though a response surface methodology is primarily aimed at the modeling of packaged devices or complex systems [11], it is hard to provide a well-established behavioral model reference. The idea of adaptive sampling is to consecutively perform the measurements that are expected to provide the most valuable information, e.g., in highly nonlinear areas or undersampled areas. Adaptive sampling allows obtaining behavioral models that outperform the empirical 
model within already 70 measurement samples [16].

In this paper, which is an extension of [16], the response surface methodology is not only compared to a CAD available empirical model, but it is also proposed as its complementary part. The novelty lies in the proper combination of the advantages provided by empirical and behavioral models, as only a few types of behavioral models are suitable to form a hybrid with good extrapolation capabilities. Due to incorporation of the response surface concepts, a direct and very efficient extraction of the proposed hybrid model is achieved.

The paper is organized as follows. In Section II the hybrid model structure is discussed with particular attention payed to the requirements for the behavioral part. Then, in Section III the model extraction procedure is explained in comparison to the classical approach of empirical model extraction using large-signal measurement data. Section IV presents the experimental setup that allows a careful and consistent comparison of the results discussed in Section V. Finally, the conclusions are drawn in Section VI.

\section{HYBRID MODEL}

The hybrid model can be formulated as follows:

$$
y(x)=e(x)+b(x),
$$

where $y$ is the value of the modeled quantity calculated for the sample $x$ using the empirical $e$ and behavioral $b$ models. Since the hybrid model should be implementable in CAD environments, $y$ should be either a current/voltage or incident/scattered wave pair. The sample $x$ can be either LSOP or the time-domain waveform, depending on the simulation set-up and model implementation.

Since (1) is very general, it allows a great level of freedom in choosing how each part of the model contributes to the overall accuracy. However, one should have in mind that the behavioral model performs poorly in extrapolation. Therefore, the hybrid model should be based mainly on a robust, wellfitted empirical part to maintain the model functionality in a wide range of input variables. At the same time, the behavioral part can be used to boost the accuracy in the region of interest, as it performs well in the interpolation tasks.

The biggest advantage of (1) is that the requirements for the interpolation accuracy of the empirical part are much more relaxed than for the accuracy of purely empirical models. Therefore, empirical model has no longer to be modified in order to improve the accuracy. As stated before, such modifications require vast user experience and insight into the device behavior. Extraction of the hybrid model allows reusage of existing tools for the extraction of the empirical part. The additional cost of hybrid model extraction and implementation is relatively small in comparison to the development or modification of the empirical model itself.

Furthermore, one can replace the expensive extraction procedures based on the multiobjective optimization of multiple model parameters with, usually less accurate, but direct extraction procedures. The difference $\Delta$ between the measurement value $\mathbf{Y}(\mathbf{X})$ and the empirical model prediction $e(\mathbf{X})$ :

$$
\Delta=\mathbf{Y}(\mathbf{X})-e(\mathbf{X})
$$

can be always approximated by a behavioral model. Of course, the better the fit of the empirical model, the less complex behavioral model is needed. However, the extraction of the behavioral part is relatively cheap in terms of user experience and expertise level, and the whole extraction method of the hybrid model can be easily automated.

Even though the proposed formulation (1) is very general, it puts specific demands on the behavioral part of the hybrid model, as described in the following Sections.

\section{A. Differentiability}

The very first requirement is that the behavioral part should be differentiable with respect to the input variables. This is needed by many optimizers and some simulators like harmonic balance analysis. Furthermore, this requirement suits better physical phenomena, in which the speed of processes is limited by the finite amount of power. Most of the behavioral models used in the microwave field meet this requirement. One of the most successful are splines, Artificial Neural Networks (ANN), and Radial Basis Functions (RBF).

\section{B. Convergence in the extrapolation region}

Since the behavioral models are purely data based, they have no or very limited extrapolation capabilities. On the contrary, empirical models usually perform well over a very broad range of different variables. As such, the hybrid model should be based on both models in the interpolation region, and only on the empirical models in the extrapolation region $(\Delta=0)$. However, one cannot simply set the response of the behavioral models to zero when entering the extrapolation region, as it will violate the first requirement of differentiability.

In theory, splines are possible, but they are not well-suited for scattered data sets generated by the adaptive sampling. Therefore, the behavioral models themselves need to ensure that their response is zero at infinity. For example, the popular ANN with sigmoid activation function cannot be used as the output layer. Instead, some types of RBFs can be employed, e.g., Gaussian and inverse multiquadratic [18]. In this work we consider interpolation Gaussian RBF models:

$$
y(x)=\sum_{i=1}^{N} w_{i} \exp \left(-\gamma\left\|x-c_{i}\right\|\right),
$$

where $w_{i}$ is the $i$-th weight, $c_{i}$ is the $i$-th sample, $\gamma$ is the shape parameter common for all the RBFs and $N$ is the number of samples. It is infinitely differentiable, and it has already been shown that it performs well in modeling of nonlinear active devices [11], [16].

\section{Extraction Procedure}

The flow diagram depicting the proposed model extraction procedure is shown in Fig. 1. The first step of the procedure consists of extracting the main parameters of the empirical model directly from measurements, without any optimization steps. Specifically, initial values of the parasitic elements can be extracted from 'cold' FET measurements with the channel pinched and the channel open [19]. Next, multibias 


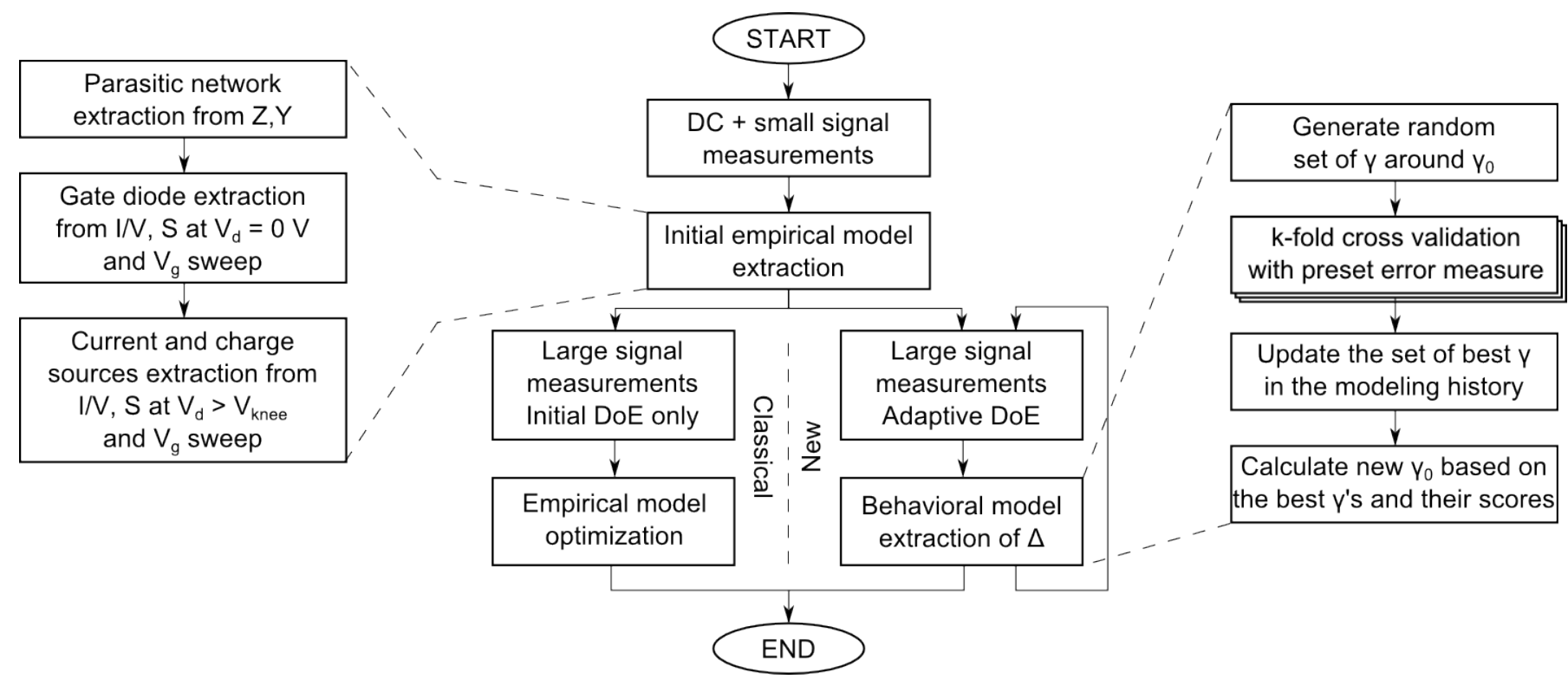

Fig. 1. Flow diagram of the model extraction procedure with optimization of the empirical model against large signal measurements (left branch), and the proposed procedure of hybrid model extraction with response surface methodology (right branch).

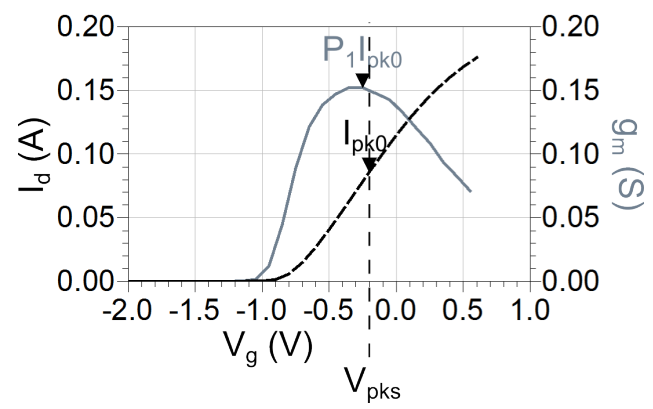

Fig. 2. Measured dc current at the drain port $I_{d}$ (black dashed line) and transconductance $g_{m}$ (gray solid line) as a function of $V_{g}$ at $V_{d}=6 \mathrm{~V}$.

Y-parameters, derived from multibias S-parameters, can be used to derive initial estimate for the nonlinear capacitances model [20]. Finally, the main parameters of the nonlinear currents can be extracted by few dc measurements in linear and saturation region. This paper follows the Angelov model extraction-procedure described in [21]. As regarding the gate Schottky junction, parameters can be extracted by keeping $V_{d}=0 \mathrm{~V}$ and sweeping $V_{g}$ to drive the junction in forward conduction. Then, the parameters of the drain current and charge sources are extracted from the $V_{g}$ sweep, while keeping the device in the saturation region $\left(V_{d}>V_{k n e e}\right)$ and cold region $V_{d}=0 \mathrm{~V}_{-}$. For example, the main parameters of the drain current and gate-source charge can be extracted as shown in Fig. 2 and Fig. 3. The other model parameters can be either set to the default values implemented in the CAD or gathered from literature [21] .

It has to be noticed that all the parameters of the initial

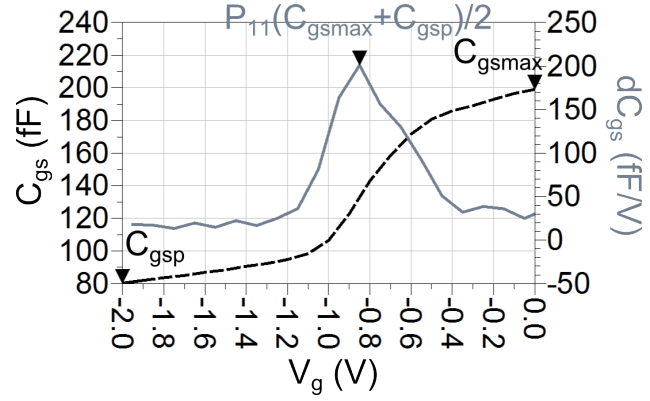

Fig. 3. $C_{g s}$ derived from multibias S-parameters at $f=2 \mathrm{GHz}$ (black dashed line) and its derivative $d C_{g s}$ (gray solid line) as a function of $V_{g}$ at $V_{d}=0 \mathrm{~V}$.

empirical model are determined directly from the data, and there is no need for optimization. Thus, the model extraction is particularly simple in implementation and evaluation [22].

In the classical approach, once initial values of the model parameters are determined, the next step consists of fitting the nonlinear currents and charges against nonlinear measurements [23]. Large signal measurements are typically gathered following the factorial Design of Experiments (DoE) [24]. In this DoE samples are placed equidistantly on the tensor product grid, i.e., samples are uniformly set along each of the input dimensions. The number of samples per dimension is referred to as the factorial DoE level. The process of optimization may be the most troublesome, as it may require user experience and supervision. Since the empirical model consists of multiple nonlinear functions, the cost function used by the optimizer has very likely multiple local optima. Moreover, the model parameters have to be constrained during optimization to a very particular ranges of values, in order to avoid unphysical or unreasonable results. Another challenge is setting the proper size of the initial DoE in large-signal 
measurements. A too small size will impede the final model accuracy, while a too large size will unduly prolong the optimization time.

Contrary to the classical approach, in the proposed method the large signal measurements are performed sequentially using adaptive sampling algorithms. For each of the LSOP samples, the corresponding response of the initial empirical model is calculated using existing CAD tools, which allows computing the difference $\Delta$ between the measurement results and empirical model prediction. Since the intermediate models of $\Delta$ serve in the adaptive sampling algorithms, the response surface methodology should focus on the regions, where the initial empirical model does not reflect the true behavior of the measured device, i.e., where the prediction error is nonlinear. In this way, the total error of the resulting hybrid model is expected to diminish very quickly with an increasing number of large-signal measurements.

The shape parameter $\gamma$ of interpolation RBF models (3) was determined in an evolutionary procedure, which is far computationally-cheaper than nonlinear optimization for $\gamma$. In each modeling iteration, 20 realizations of $\gamma$ are generated randomly with normal distribution (trimmed to $[0.5,5]$ range) around a given starting value $\gamma_{0}$. Then, a five-fold crossvalidation error-measure of root relative square error is calculated and stored for each $\gamma$ [9]. If the gamma is set, the model parameters $w_{i}$ are unique solution to a linear system of equations [18]. It can be solved in a single algebraic operation instead of relatively expensive iterative optimization, which is used in example in ANN extraction [25]. In the previous works [11], [16], we have also included polynomial part in the RBF models to improve their interpolation performance. However, polynomials cannot be used for the hybrid models, as they do not converge to 0 at the infinity, and they will impede the extrapolation capabilities. $\gamma_{h}$ corresponding to the best $H=20$ scores $s_{h}$ in the whole modeling history are used to calculate new starting value $\gamma_{0}$ for the next modeling iteration:

$$
\begin{array}{r}
\gamma_{0}=\frac{\sum_{h=1}^{H} \gamma_{h} c_{h}}{\sum_{h=1}^{H} c_{h}}, \\
c_{h}=1-\frac{s_{h}}{\|\mathbf{S}\|},
\end{array}
$$

where $\mathbf{S}$ is a vector of $s_{h}$. The $\gamma$ corresponding to the best measure score in the history was used to extract the final model.

\section{EXPERIMENTAL SETUP}

In order to evaluate the proposed method, the measurement system shown in Fig. 4 was set up. It consists of a Source Measure Unit (SMU), which sets the bias voltages and reads the corresponding currents, and a Nonlinear Vector Network Analyzer (NVNA) that is responsible for collecting small signal and large signal data. The Device Under Test (DUT) was a six-finger GaAs pHEMT manufactured by TriQuint with gate dimensions $0.15 \mu m \times 60 \mu m$ (same technology, but different device than in [16]). Much attention was paid to the proper calibration, i.e., dc calibration of the lead resistances, TRL

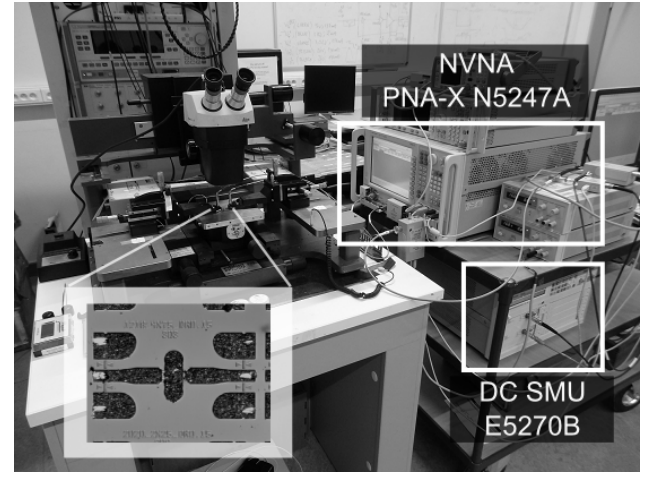

Fig. 4. Picture of the measurement setup and the device-under-test.

TABLE I

INITIAL VALUES OF THE EMPIRICAL MODEL PARAMETERS.

\begin{tabular}{|c|c||c|c||c|c|}
\hline$I_{p k 0}(\mathrm{~mA})$ & 80 & $R_{g}(\Omega)$ & 2.539 & $C_{g s p}(\mathrm{fF})$ & 80 \\
\hline$P_{1}$ & 1.918 & $R_{d}(\Omega)$ & 1.245 & $C_{g s 0}(\mathrm{fF})$ & 162 \\
\hline$P_{2}$ & 0 & $R_{s}(\Omega)$ & 0.139 & $C_{g d p}(\mathrm{fF})$ & 41.94 \\
\hline$P_{3}$ & 0.07 & $L_{g}(\mathrm{pH})$ & 28.1 & $C_{g d 0}(\mathrm{fF})$ & 66.5 \\
\hline$V_{p k s}(\mathrm{~V})$ & -0.2 & $L_{d}(\mathrm{pH})$ & 20.43 & $P_{10}, P_{40}$ & 1.5 \\
\hline$D V_{p k s}(\mathrm{~V})$ & 0.3 & $L_{s}(\mathrm{pH})$ & 4.48 & $P_{11}, P_{41}$ & 1.7 \\
\hline$B_{1}$ & 1 & $C_{d s}(\mathrm{fF})$ & 77 & $P_{20}$ & -0.76 \\
\hline$B_{2}$ & 3 & $V_{j}(\mathrm{~V})$ & 0.55 & $P_{21}, P_{31}$ & 0.2 \\
\hline$\alpha_{R}$ & 0.1 & $I_{j}(\mu \mathrm{A})$ & 474 & $P_{30}$ & 0 \\
\hline$\alpha_{S}$ & 1.4 & $P_{g}$ & 14.3 & & \\
\hline
\end{tabular}

calibration with deembedding structures. Inaccurate measurements can perturb the empirical model extraction, and thus bias the comparison results.

During the initial stage of the extraction procedure shown in Fig. 1 the dc voltages were swept as follows: gate voltage $V_{g} \in[-2,0.6] \mathrm{V}$ with $0.1 \mathrm{~V}$ step, and drain voltage $V_{d} \in[0,9] \mathrm{V}$ with $0.2 \mathrm{~V}$ step. In order to protect the DUT, the power constraint was set to $0.3 \mathrm{~W}$. For each of the bias points S-parameters were collected over the frequency range $f_{0} \in[0.1,40] \mathrm{GHz}$ with $0.1 \mathrm{GHz}$ step. The initial set of empirical model parameters is shown in Tab. I. In the second stage of both procedures (standard and proposed), large signal measurements were performed as a function of the following input variables: gate voltage $V_{g} \in[-1.3,-0.7] \mathrm{V}$, drain voltage $V_{d} \in[0,6] \mathrm{V}$, and input power $P_{\text {in }} \in[-10,3] \mathrm{dBm}$. The fundamental frequency was $f_{0}=2 \mathrm{GHz}$. The output variables were the dc currents $I_{g}, I_{d}$ and scattered traveling voltage waves $b_{g}, b_{d}$ at the first three harmonics of $f_{0}$ acquired at gate and drain ports, respectively. As the interpolation RBF models considered in this work support only real output quantities, 14 quantities were modeled in total.

For the classical approach, the large signal measurements were acquired according to the factorial DoE, whose level was swept from 2 to 10 (from 8 to 1000 samples). This allowed us investigating how the empirical model optimization process is affected by the amount of training data. The model optimization was performed in Keysight ADS v. 2013 using combination of random and gradient optimizers with 2000 iterations as a stop criterion. All the calculations in this paper 
were done on the same machine (i7-3610QM @ $2.3 \mathrm{GHz}$ ), in order to have a consistent comparison..

All the experiments were automated and the controlling software (Matlab) was interfaced with a circuit simulator (Keysight ADS v. 2013) enabling harmonic-balance in order to compute the response of the empirical model. The work is based on a modified version of Matlab's SUrrogate MOdeling Toolbox, which implements response surface methodology [26]. Among others, SUMO Toolbox enables adaptive sampling and model-extraction techniques.

The adaptive sampling algorithm was a mixture of Voronoi (space exploration), LOLA (nonlinearity exploitation), and model-error minimization methods [11], [27], [28]. In order to combine the adaptive sampling methods, the scores of Voronoi and LOLA rankers were multiplied. As it is impossible to do so with the error-based sampling algorithm, random selection of the sampling method was performed [11]. For each requested sample, probability of choosing Voronoi-LOLA mixture was $70 \%$, and error-based algorithm was $30 \%$.

In order to have comparable results to the classical approach, the final stop criterion was the evaluation of 512 samples, which corresponds to the factorial DoE level eight. It must be emphasized though that the stop criterion in a response surface methodology can usually be set by an errorrelated threshold value. This prevents oversampling, which might result in model overfitting.

Two experiments with hybrid models were conducted. In the first one, the empirical part was the coarse, not-optimized empirical model [17] described by Tab. I. In the second hybrid model, its empirical part was optimized against 1000 large signal measurements. This allowed assessing the contribution of each of the model parts to the model accuracy.

In addition to the large signal measurements collected for both methods, two independent test sets were collected. The first data set consisting of 3000 large signal measurement samples in the same input variables ranges as for the measurements for the model extraction. This dataset was used as a validation set for interpolation capabilities. Since the whole experiment took considerable amount of time (two days), part of this test set $(D=512$ samples in total) was remeasured between each of the experiment stages $(R=5$ repetitions in total). This allowed assessing the drifts of the experimental set-up:

$$
\text { drift }=\frac{1}{D} \sum_{i=1}^{D}\left(\max _{r \in[2, R]}\left|m_{r}\left(t_{i}\right)-m_{1}\left(t_{i}\right)\right|\right),
$$

where $m_{r}$ is the $r$-th repetition of the measurement for sample $t_{i}$.

The second test set consisting of 3000 samples was meant for extrapolation assessment. Therefore, the input variables ranges were extended as follows: $V_{g} \in[-2,-0.2] \mathrm{V}, V_{d} \in$ $[0,9] \mathrm{V}, P_{i n} \in[-15,8] \mathrm{dBm}$. This test set was constrained by the maximum dissipated power $P_{\text {diss }}<0.3 \mathrm{~W}$, maximum instantaneous gate voltage $V_{\text {gmax }}(t)<0.4 \mathrm{~V}$, and maximum instantaneous drain voltage $V_{d \max }(t)<10.5 \mathrm{~V}$. The region common with the interpolation test set was excluded. The input variables samples in both validation sets (interpolation and extrapolation) were generated randomly with uniform distribution.
The test sets were employed to calculate the error metrics: Mean Absolute Error (MAE), Mean Square Error(MSE), and Mean Relative Error (MRE). The proposed hybrid model is meant for design applications, thus, the Mean Absolute Error is shown in this paper, as it shows the average performance across the whole input variables' space. However, the other metrics are also monitored, as large MSE values indicate the presence of outliers, and large MRE values suggests that the model has problems with prediction of small quantities.

\section{RESUlTS AND DISCUSSION}

\section{A. Interpolation}

Fig. 5 shows MAE of the scattered waves as a function of the number of large-signal samples used to train the model. The hybrid models show superior performance over the purely empirical model for all the modeled quantities irrespectively to the error metric (MAE, MSE, MRE).. The errors become smaller already within the first 27 samples. In comparison to the purely RBF models, the hybrids also show better performance for all the quantities except for the scattered wave at the gate side at the third harmonic, as shown in Fig. 5c.

This is caused by inaccurate empirical base, which has to replace the polynomial part of a standard RBF model. Coarse empirical model was directly extracted from the S-parameters and dc measurements, and as is does not account for the nonlinearities manifested in large-signal measurements. Moreover, the higher order derivatives, which serve to extract the nonlinear drain sources, are prone to the measurement noise. Optimized empirical models also fail in accurate prediction of the quantities at higher harmonics since they were optimized to the large-signal data using MSE.

Contrary, the purely RBF models are much better in modeling of highly nonlinear quantities at higher harmonics than the relatively linear ones at the fundamental frequency. This is caused by the always-changing shape of the basis function, which is not suitable for linear tasks. It is particularly pronounced in Fig. 5a, where an over-fitting occurs for more than 125 samples. More basis functions only causes the response to be more corrugated, while the true device behavior is linear. This is consistent with the results presented in [11], [16].

It can be seen that the accuracy of hybrid models is greater than the sole RBF or empirical models, irrespectively on the empirical base of both hybrids. It takes less than only 27 large signal samples using adaptive sampling to gain smaller MAE than the pure empirical model optimized with 1000 large signal measurements. Furthermore, the error performance is very similar for both hybrids, and the employment of the optimized empirical part improves the results very slightly. Thus, the optimization step of the empirical model can be omitted while building a hybrid model.

For the sake of experiment sanity, the modeling errors were compared to the drifts levels gathered in Tab. II. It can be noticed that the error level is higher than the drift level, even after measurements of more than 12000 large-signal samples including the extrapolation region, and the total time of the 


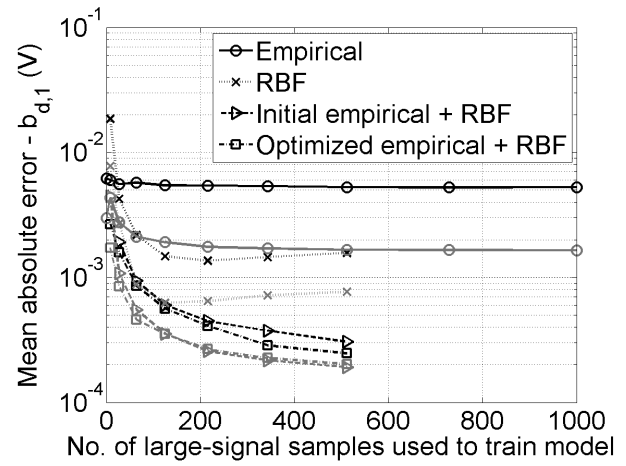

(a)

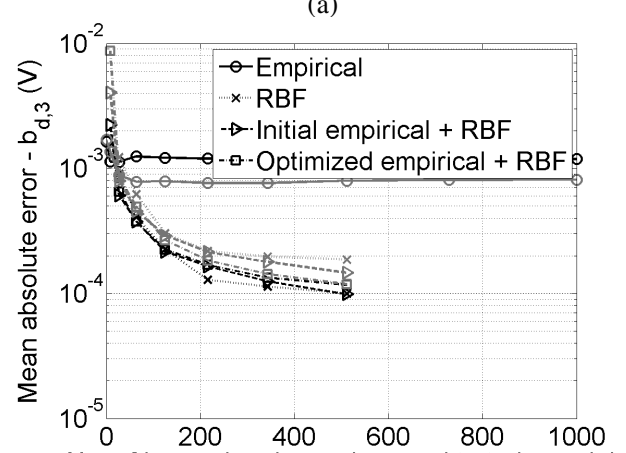

(b)

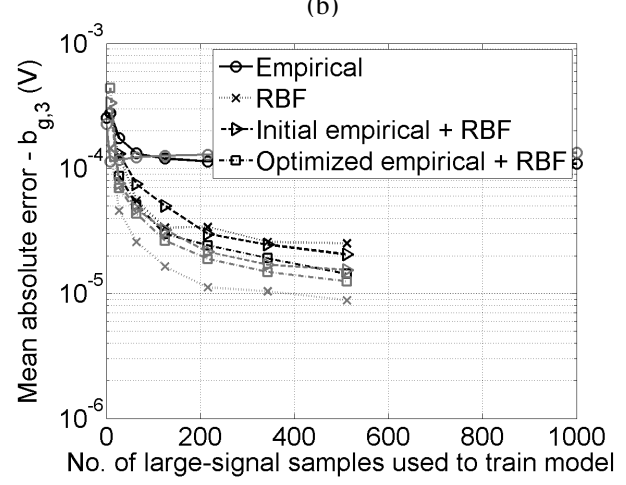

(c)

Fig. 5. Mean absolute error for different models as a function of the number of samples used to train the model. Real parts - black lines, imaginary parts - gray lines. (a) - first harmonic of scattered wave at the HEMT drain port; (b) - third harmonic of scattered wave at the HEMT drain port; (c) - third harmonic of scattered wave at the HEMT gate port.

TABLE II

DRIFTS LEVELS.

\begin{tabular}{|c||c|c||c||c|c|}
\hline Quantity & Real & Imaginary & Quantity & Real & Imaginary \\
\hline \hline$I_{g}(\mathrm{~A})$ & $2.90 \mathrm{e}-08$ & & $I_{d}(\mathrm{~A})$ & $1.94 \mathrm{e}-05$ & \\
\hline$b_{g, 1}(\mathrm{~V})$ & $4.30 \mathrm{e}-05$ & $2.10 \mathrm{e}-05$ & $b_{d, 1}(\mathrm{~V})$ & $1.58 \mathrm{e}-04$ & $5.18 \mathrm{e}-05$ \\
\hline$b_{g, 2}(\mathrm{~V})$ & $5.15 \mathrm{e}-06$ & $7.64 \mathrm{e}-06$ & $b_{d, 2}(\mathrm{~V})$ & $5.59 \mathrm{e}-05$ & $4.60 \mathrm{e}-05$ \\
\hline$b_{g, 3}(\mathrm{~V})$ & $2.20 \mathrm{e}-06$ & $2.17 \mathrm{e}-06$ & $b_{d, 3}(\mathrm{~V})$ & $1.38 \mathrm{e}-05$ & $1.38 \mathrm{e}-05$ \\
\hline
\end{tabular}

experiment exceeding two days. Thus, measurements can be perceived as consistent and the errors originate rather in the models than in the non-stationarity of the measurement system.

In order to check if the modeling performance is uniform in the input variables space, the sum of absolute errors for all the modeled quantities was calculated in three bins per input variable. The best models were taken into consideration, i.e.,

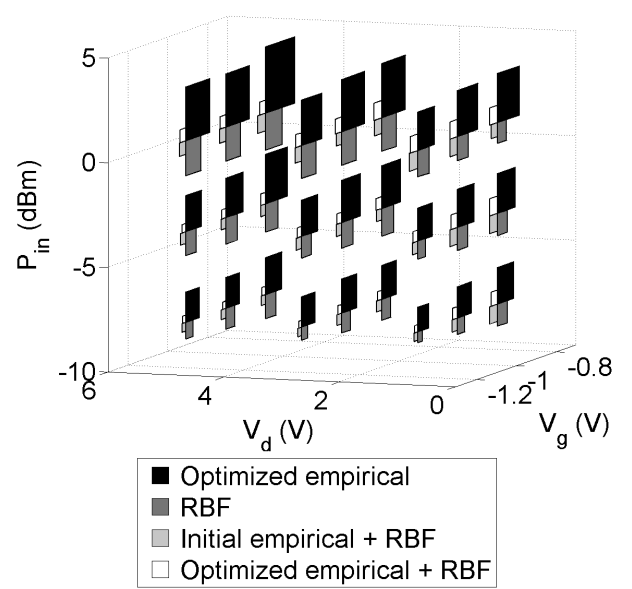

Fig. 6. Absolute error distribution for the considered models in a $3 \times 3 \times 3$ bin grid. The area of each square is proportional to the total absolute error of the corresponding model. The subsequent squares are placed clockwise starting from top-right around the centers of the bins.

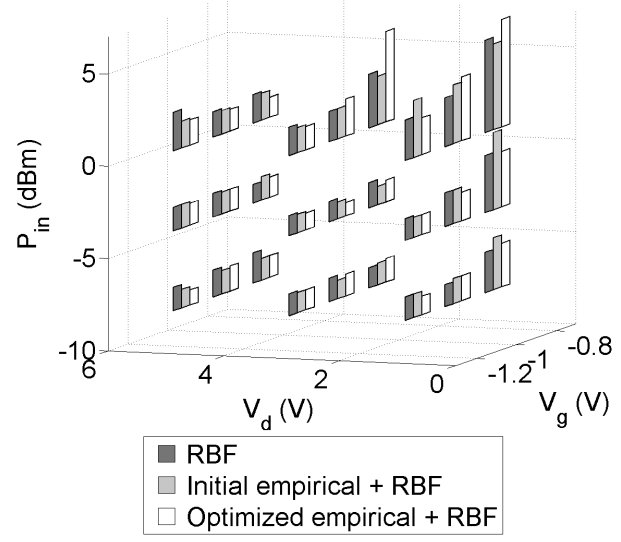

Fig. 7. Samples distribution for models using adaptive sampling in a $3 \times 3 \times 3$ bin grid. The height of each rectangle is proportional to the number of samples in the corresponding bin.

the empirical model optimized with 1000 large signal samples, and RBF-based with 512 samples. The results are reported in Fig. 6. It can be clearly seen that the error relationships between the models, which are depicted in Fig. 5, are maintained in the whole space of input variables. Thereby, the hybrid models are suitable for describing various phenomena present in the DUT, and not only particular ones. For pure empirical and pure behavioral models the error is highest in the area corresponding to high $V_{g}, P_{i n}$, and $V_{d}$ values, where the values of the modeled quantities are largest. At the same time, for hybrid models the most erroneous area is shifted towards low $V_{d}$ values, where the DUT shows bigger nonlinearity than in the saturation region.

To check how the error is supported by adaptive sampling, the sample distributions were calculated in the same bins as for the error distribution. The results are shown in Fig. 7. It can be noticed that the adaptive sampling places considerably more samples in the highly nonlinear region (LOLA algorithm), while the rest of the input space is sampled evenly (Voronoi 


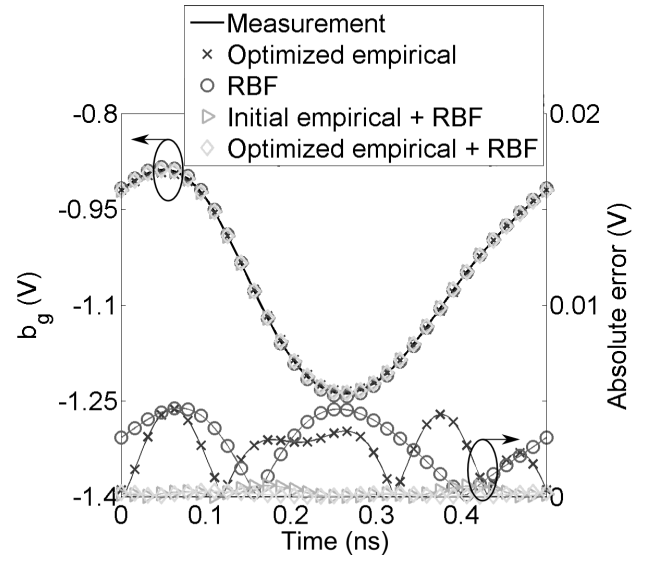

(a)

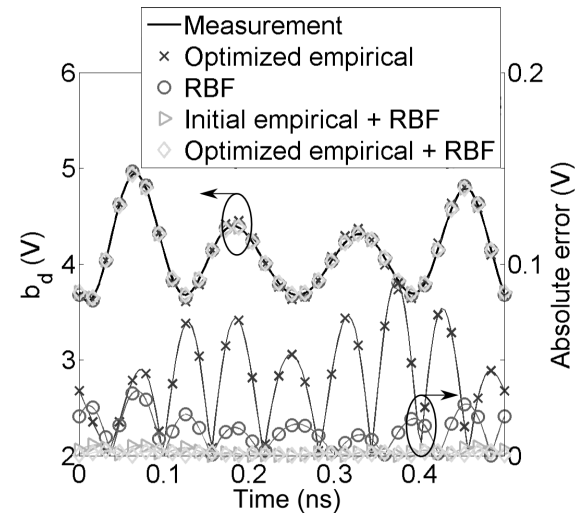

(b)

Fig. 8. Time-domain waveforms of the scattered waves for the considered models in comparison to the measurement result. The MAE for the considered measurement sample is the closest value to the median absolute error for the hybrid consisting of RBF and initial empirical models. (a) - scattered waves at the HEMT gate port; (b) - scattered waves at the HEMT drain port.

algorithm). However, the empirical core of the hybrid model allows focusing more on the more nonlinear regions, e.g., optimized empirical, RBF hybrid vs. pure RBF. Notwithstanding the small differences in distributions, the better error performance of the hybrid models in comparison to pure RBF model is due to employment of the good empirical base for hybrid models.

Finally, in order to give a better feeling of the aggregated error measure MAE, the typical performance of the hybrid models was investigated in Fig. 8. The MAE for the considered measurement sample is the closest value to the median error value for the hybrid consisting of RBF and initial empirical models. One can perceive that all the models retain similarity to the measured waveform. The maximum error for the optimized empirical model equals to $0.0045 \mathrm{~V}$ for $b_{g}$ and $0.091 \mathrm{~V}$ for $b_{d}$. RBF shows the same error for $b_{g}$, but for $b_{d}$ the maximum error is lower, $0.033 \mathrm{~V}$. However, the hybrid models perform much better irrespectively to the DUT's side. For $b_{g}$ the error does not exceed $0.0005 \mathrm{~V}$ and $0.0003 \mathrm{~V}$ for the hybrid with coarse empirical model and with optimized empirical model, respectively. For the $b_{d}$ the corresponding error values are $0.002 \mathrm{~V}$ and $0.005 \mathrm{~V}$. The results are consistent with Fig. 5.

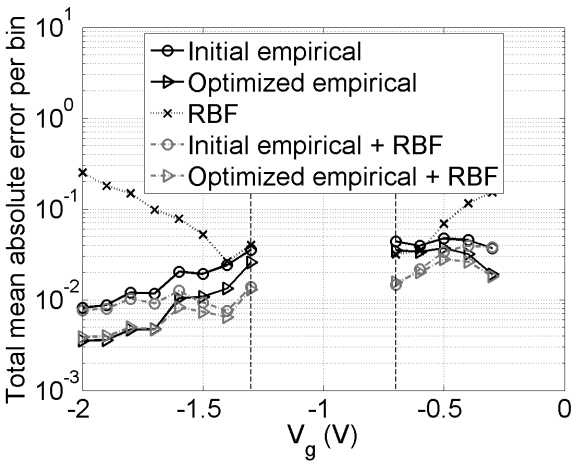

(a)

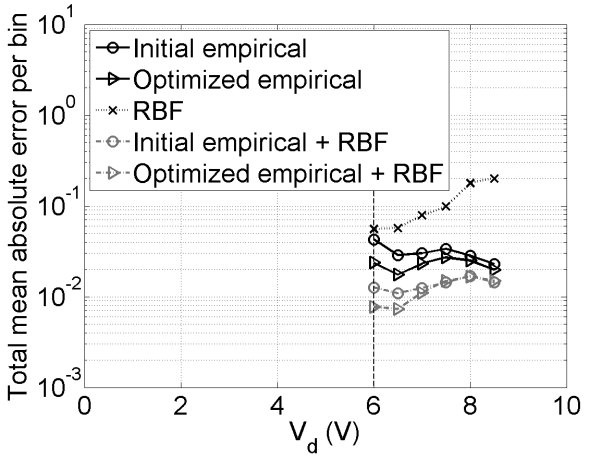

(b)

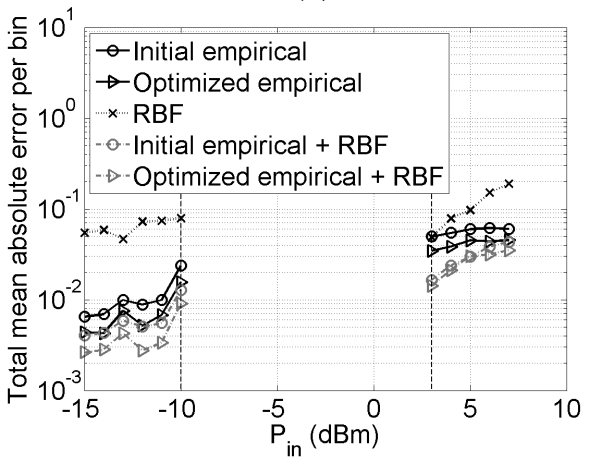

(c)

Fig. 9. Total mean absolute error of the models in the extrapolation regions per bin along different input variables: (a) - gate voltage $V_{g}$, (b) - drain voltage $V_{d}$, (c) - input power $P_{i n}$. The vertical dashed lines delimit the interpolation and extrapolation regions.

\section{B. Extrapolation}

After the assessment of the interpolation capabilities of the considered models, their extrapolation performance was evaluated using the extrapolation test set. The total error for all the quantities predicted by different model types was calculated in $1 \mathrm{D}$ uniform bins along the input variables. The results are shown in Fig. 9. As expected, pure behavioral RBF model has the worst extrapolation performance. At the same time, the hybrid models outperform even the pure empirical ones in all the extrapolation regions. This proves that the assumptions of differentiability and convergence make sense. Since the physical phenomena are differentiable, the resulting characteristics of the DUT are not expected to be very abrupt. As the error of the hybrid models is small in the interpolation region, it should also remain smaller in the close proximity to the interpolation region due to differentiability. In larger distance from the 


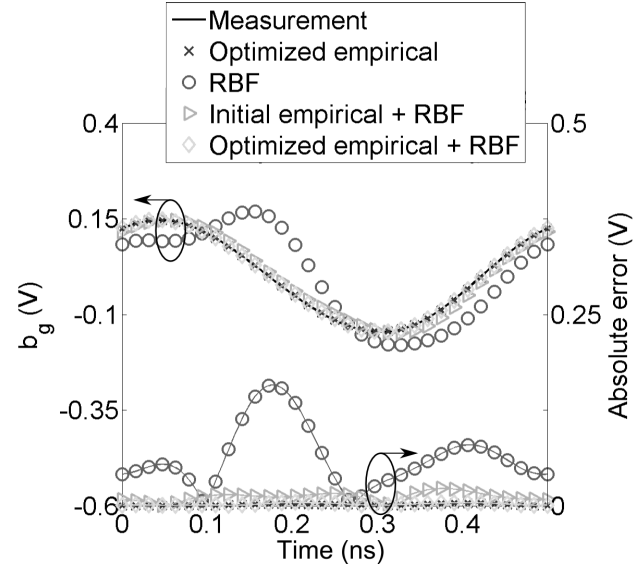

(a)

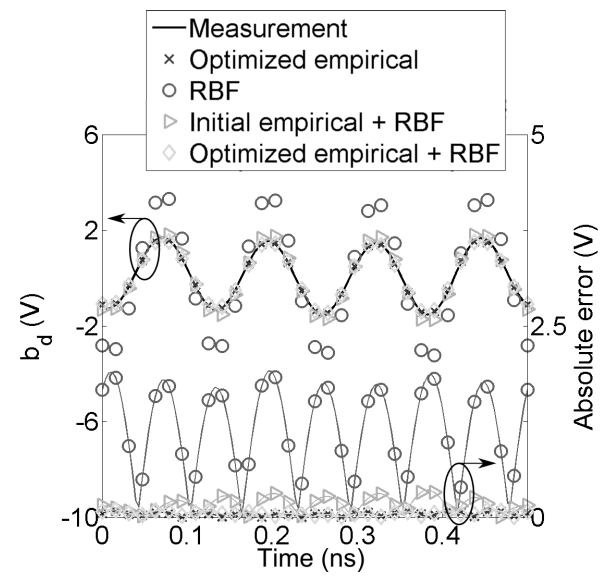

(b)

Fig. 10. Time-domain waveforms of the scattered waves for the considered models in comparison to the measurement result. The sample was chosen to lie deep in the extrapolation region: $V_{g}=0.20 \mathrm{~V}, V_{d}=2.79 \mathrm{~V}, P_{\text {in }}=$ $-5.06 \mathrm{dBm}$. (a) - scattered waves at the HEMT gate port; (b) - scattered waves at the HEMT drain port.

interpolation region, the models profit exponentially from their empirical base, as the behavioral contribution decays with the euclidean norm $\left\|x-c_{i}\right\|$ (3). Therefore, the hybrid model response converges to the response of its empirical part. It is particularly visible in Fig. 9a, where the extrapolation region is relatively wide in comparison to the interpolation one. Concluding, the extrapolation performance relies mainly on the fitting of the empirical part, as can be seen when comparing the hybrid models with different empirical bases.

Example waveforms for the sample lying deep in the extrapolation region $\left(V_{g}=0.20 \mathrm{~V}, V_{d}=2.79 \mathrm{~V}, P_{i n}=-5.06 \mathrm{dBm}\right)$ are shown in Fig. 10. It can be seen that the error is inversely proportional to the contribution of the empirical model. Pure RBF models show the worst performance with error reaching $0.16 \mathrm{~V}$ for $b_{g}$ and $1.86 \mathrm{~V}$ for $b_{d}$. Addition of non-optimized empirical model allows to predict the waveforms significantly better with maximum error of $0.02 \mathrm{~V}$ and $0.35 \mathrm{~V}$ for $b_{g}$ and $b_{d}$, respectively. The best extrapolation capabilities were achieved for optimized empirical model and its hybrid, for which the $b_{g}$ errors are smaller than $0.005 \mathrm{~V}$ for $b_{g}$ and $0.007 \mathrm{~V}$ for $b_{d}$. This can be explained by the fact that the response of the hybrid model is almost completely based on its empirical part when

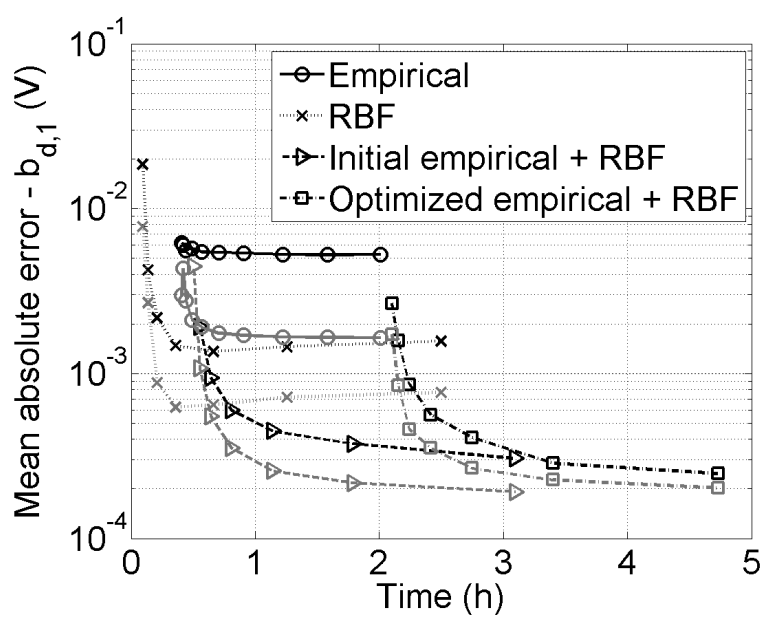

Fig. 11. Mean absolute error for different models as a function of measurement and computational time. Real parts - black lines, imaginary parts - gray lines.

it comes to significant extrapolation. The results are consistent with Fig. 9.

\section{Complexity}

Last but not least, the computational complexity of the methods was investigated. Thereby, the same MAE figures as in Fig. 5, but as a function of the time needed to reach the corresponding error levels were plotted. The results for $b_{d, 1}$ are shown in Fig. 11. The benefit of incorporating RBF models with adaptive sampling is now even more pronounced. The initial dc and small-signal measurements and extraction of the initial empirical model took 24 minutes in total. Within this time one can already extract a much more accurate pure RBF model with 141 samples. However, it takes only additional 8 minutes (27 samples) to extract a hybrid that is more accurate than the pure empirical model extracted with 1000 samples. This is true for all the measured quantities. After next 5 minutes (37 minutes and 52 large signal samples in total) hybrid model with coarse empirical part becomes better than the pure behavioral model with 203 samples. Similar performance was achieved for other measured quantities, except the ones for the third harmonic, for which pure RBF models show similar interpolation error to the hybrid models. The measurements and the optimization of the empirical model with 1000 samples took 120 minutes in total. In this time, one can extract hybrid model with coarse empirical part and RBF part with 370 large signal samples, which has more than one order of magnitude smaller error for $b_{d, 1}$. Unless the purpose of the model extraction is providing insight of the device phenomena, the proposed hybrid is a good candidate for replacement of the lengthy empirical model optimization in design applications.

However, it must be emphasized that the computational complexity of the response surface technology is higher than the sole optimization complexity of the empirical model. It can be seen that the optimization with 1000 samples takes less time that achieving 512 samples in response surface technology. This is related to the intermediate modeling steps, 
as well as the sampling algorithms complexity. Nevertheless, the response surface methodology aims at maximizing the information gain from the initial samples, where the complexityrelated issues are less cumbersome.

\section{CONCLusions}

In this paper, we have discussed a method for the direct extraction of empirical-behavioral hybrid models using adaptive sampling. The resulting model is particularly meant for the design applications. The hybrid combines robustness of the empirical part with interpolation accuracy of the behavioral part. Employment of the response surface methodology enabled full automation of the extraction procedure. The adaptive sampling allowed minimizing the number of samples required to achieve accurate model. The resulting compactness of the hybrid model is important criterion in the model evaluation and distribution. The model was based on the measurements of a $0.15 \mu \mathrm{m}$ GaAs HEMT. The Angelov empirical base of the hybrid model was extracted directly from dc and small-signal measurements. The behavioral part of the hybrid constituted of interpolation Radial Basis Function model with Gaussian basis function, which assures differentiability and convergence of the hybrid to its empirical part in the extrapolation region. The hybrid model allowed achieving a better interpolation performance within the first 27 samples comparing to the empirical model optimized with 1000 samples. In design applications, short extraction time and straightforward procedure makes the hybrid model a good replacement for the timeconsuming and experience-demanding optimization procedure of the pure empirical model. At the same time, the hybrid persists the extrapolation performance similar to the optimized empirical model, which is an order of magnitude better than the performance of the pure behavioral model.

\section{ACKNOWLEDGMENTS}

P. Barmuta would like to thank prof. Ilcho Angelov for valuable discussions.

\section{REFERENCES}

[1] S. Albahrani, J. G. Rathmell, and A. E. Parker, "Characterizing drain current dispersion in GaN HEMTs with a new trap model," in Europ. Microw. Conf. (EuMC). IEEE, 2009, pp. 1692-1695.

[2] P. Cabral, J. Pedro, and N. Carvalho, "Nonlinear device model of microwave power GaN HEMTs for high power-amplifier design," $M i$ crowave Theory and Techniques, IEEE Transactions on, vol. 52, no. 11, pp. 2585-2592, Nov 2004.

[3] D. Root, "Future device modeling trends," IEEE Microw. Mag., vol. 13, no. 7, pp. 45-59, Nov 2012.

[4] P. Roblin, D. Root, J. Verspecht, Y. Ko, and J. Teyssier, "New trends for the nonlinear measurement and modeling of high-power RF transistors and amplifiers with memory effects," IEEE Trans. Microw. Theory Techn., vol. 60, no. 6, pp. 1964-1978, June 2012.

[5] J. Wood, D. Root, and N. B. Tufillaro, "A behavioral modeling approach to nonlinear model-order reduction for RF/microwave ICs and systems," IEEE Trans. Microw. Theory Techn., vol. 52, no. 9, pp. 2274-2284, Sept 2004.

[6] D. Ribeiro, P. Cruz, and N. Borges Carvalho, "Synchronous oversampled measurements for the extraction of mixed-signal behavioral models in digital to analog integrated transmitters," IEEE Trans. Microw. Theory Techn., vol. 62, no. 12, pp. 3183-3192, Dec 2014.

[7] P. Cruz and N. Carvalho, "Wideband behavioral model for nonlinear operation of bandpass sampling receivers," IEEE Trans. Microw. Theory Techn., vol. 59, no. 4, pp. 1006-1015, April 2011.
[8] W. Demenitroux, C. Maziere, E. Gatard, S. Dellier, M. Campovecchio, and R. Quere, "Multiharmonic volterra model dedicated to the design of wideband and highly efficient GaN power amplifiers," IEEE Trans. Microw. Theory Techn., vol. 60, no. 6, pp. 1817-1828, June 2012.

[9] A. Forrester, A. Sobester, and A. Keane, Engineering Design via Surrogate Modelling: A Practical Guide. Wiley, 2008.

[10] D. Motgomery, Design and Analysis of Experiments, 6th Edition. Wiley, 2004.

[11] P. Barmuta, F. Ferranti, G. Gibiino, A. Lewandowski, and D. Schreurs, "Compact behavioral models of nonlinear active devices using response surface methodology," IEEE Trans. Microw. Theory Techn., vol. 63, no. 1, pp. 56-64, Jan 2015

[12] I. Angelov, N. Rorsman, J. Stenarson, M. Garcia, and H. Zirath, "An empirical table-based fet model," IEEE Trans. Microw. Theory Techn., vol. 47, no. 12, pp. 2350-2357, Dec 1999.

[13] A. Raffo, G. Bosi, V. Vadala, and G. Vannini, "Behavioral modeling of GaN FETs: A load-line approach," IEEE Trans. Microw. Theory Techn., vol. 62, no. 1, pp. 73-82, Jan 2014.

[14] A. Santarelli, D. Niessen, R. Cignani, G. Gibiino, P. Traverso, C. Florian, D. Schreurs, and F. Filicori, "GaN FET nonlinear modeling based on double pulse I/ V characteristics," IEEE Trans. Microw. Theory Techn., vol. 62, no. 12, pp. 3262-3273, Dec 2014.

[15] G. Crupi, D. Schreurs, A. Caddemi, I. Angelov, R. Liu, M. Germain, and W. De Raedt, "Combined empirical and look-up table approach for non-quasi-static modelling of GaN HEMTs," in 9th Int. Conf. on Telecommunication in Modern Satellite, Cable, and Broadcasting Services, Oct 2009, pp. 40-43.

[16] P. Barmuta, G. Avolio, F. Ferranti, A. Lewandowski, and D. Schreurs, "Large-signal modeling of on-wafer microwave transistors based on response surface methodology," in Int. Microw. Symp., May 2015, 3 p.

[17] I. Angelov, H. Zirath, and N. Rosman, "A new empirical nonlinear model for HEMT and MESFET devices," IEEE Trans. Microw. Theory Techn., vol. 40, no. 12, pp. 2258-2266, 1992.

[18] M. D. Buhmann, "Radial basis functions," Acta Numerica 2000, vol. 9, pp. $1-38,2000$.

[19] G. Crupi, D. Schreurs, and A. Caddemi, "On the small signal modeling of advanced microwave FETs: A comparative study," Int. J. of RF and Microw. Computer-Aided Eng., no. 5, pp. 417-425, May 2008.

[20] G. Avolio, A. Raffo, I. Angelov, V. Vadala, G. Crupi, A. Caddemi, G. Vannini, and D. Schreurs, "Millimeter-wave FET nonlinear modelling based on the dynamic-bias measurement technique," IEEE Trans. Microw. Theory Techn., vol. 62, no. 11, pp. 2526-2537, Nov 2014

[21] G. Crupi and D. Schreurs, Microwave de-embedding: from theory to applications. Academic Press, 2013.

[22] D. Root, J. Xu, F. Sischka, M. Marcu, J. Horn, R. Biernacki, and M. Iwamoto, "Compact and behavioral modeling of transistors from NVNA measurements: New flows and future trends," in Custom Integrated Circuits Conference (CICC), Sept 2012, pp. 1-6.

[23] I. Angelov, M. Thorsell, D. Kuylenstierna, G. Avolio, D. Schreurs, A. Raffo, and G. Vannini, "Hybrid measurement-based extraction of consistent large-signal models for microwave FETs," in Europ. Microw. Conf. (EuMC), Oct 2013, pp. 267-270.

[24] T. J. Santner, B. J. Williams, and W. I. Notz, The Design and Analysis of Computer Experiments. Springer, 2003.

[25] J. Xu, R. Jones, S. Harris, T. Nielsen, and D. Root, "Dynamic FET model - DynaFET - for GaN transistors from NVNA active source injection measurements," in Int. Microw. Symp., June 2014, pp. 1-3.

[26] D. Gorissen, K. Crombecq, I. Couckuyt, T. Dhaene, and P. Demeester, "A surrogate modeling and adaptive sampling toolbox for computer based design," J. of Mach. Learning Res., vol. 11, pp. 2051-2055, 2010.

[27] K. Crombecq, D. Gorissen, D. Deschrijver, and T. Dhaene, "A novel hybrid sequential design strategy for global surrogate modeling of computer experiments," SIAM J. on Scientific Computing, vol. 33, no. 4, pp. 1948-1974, 2011.

[28] K. Crombecq, L. De Tommasi, D. Gorissen, and T. Dhaene, "A novel sequential design strategy for global surrogate modeling," in Proc. of the Winter Simulation Conf. (WSC), 2009, pp. 731-742. 